\title{
Engaging pictograms! A Methodology for Graphic Design in enhancing Player Engagement
}

\author{
As Applied to the Design of a Serious Game for Nepalese Women with Low Literacy \\ Delphine Soriano \\ UCL Institute for Risk and Disaster \\ Reduction \\ London, United Kingdom \\ d.soriano@ucl.ac.uk \\ Sonja Mueller \\ UCL Institute for Risk and Disaster \\ Reduction \\ London, United Kingdom \\ sonja.mueller.14@ucl.ac.uk \\ Maureen Fordham \\ UCL Institute for Risk and Disaster \\ Reduction \\ London, United Kingdom \\ m.fordham@ucl.ac.uk \\ Abriti Arjyal and Sushil Baral \\ Health Research and Social \\ Development \\ Kathmandu, Nepal \\ abriti.arjyal@herdint.com \\ sushil.baral@herdint.com \\ Gareth J. Hearn \\ Hearn Geoserve Ltd \\ United Kingdom \\ gareth@hearngeoserve.co.uk \\ Virgine Le Masson \\ Overseas Development Institute \\ London, United Kingdom \\ v.lemasson@odi.org.uk \\ Andrei Boscor \\ UCL Institute for Risk and Disaster \\ Reduction \\ London, United Kingdom \\ andrei.boscor.14@ucl.ac.uk \\ Naomi M Saville \\ UCL Institute for Global Health \\ London, United Kingdom \\ n.saville@ucl.ac.uk \\ Patty Kostkova \\ UCL Institute for Risk and Disaster \\ Reduction \\ London, United Kingdom \\ p.kostkova@ucl.ac.uk
}

\begin{abstract}
In the graphic design of serious games, player engagement is an important consideration. We propose a new approach towards aiding the graphic designer to consider the major factors relevant to player engagement. This article describes a method for creating effective graphical content for serious games that takes into account the impact of complex pictograms on player engagement and on the learning process. We show how we applied our method to the design of a serious game for mobile phones aimed at Nepalese women in rural areas with low literacy skills. Initial results from case study suggest that our method helps designers to improve the design and the logic behind their use of imagery to the extent where the need to use text in the game's user interface was removed.
\end{abstract}

\section{CCS CONCEPTS}

- Human-centered computing $\rightarrow$ Graphical user interfaces; HCI theory, concepts and models; • Applied computing $\rightarrow$ E-learning;

\section{KEYWORDS}

method; engagement; graphic design; pictogram; visual complexity; abstraction; serious game; e-learning

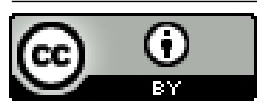

This work is licensed under a Creative Commons Attribution International 4.0 License.

DH'18, April 23-26, 2018, Lyon, France

(c) 2018 Copyright held by the owner/author(s)

ACM ISBN 978-1-4503-6493-5/18/04.

https://doi.org/10.1145/3194658.3194673
ACM Reference Format:

Delphine Soriano, Abriti Arjyal and Sushil Baral, Andrei Boscor, Sonja Mueller, Gareth J. Hearn, Naomi M Saville, Maureen Fordham, Virgine Le Masson, and Patty Kostkova. 2018. Engaging pictograms! A Methodology for Graphic Design in enhancing Player Engagement: As Applied to the Design of a Serious Game for Nepalese Women with Low Literacy. In DH'18: 2018 International Digital Health Conference, April 23-26, 2018, Lyon, France. ACM, New York, NY, USA, 5 pages. https://doi.org/10.1145/3194658.3194673

\section{INTRODUCTION}

Player engagement or motivation appears to be linked to the learning process within a game [23]. Feeling engaged during a serious game playing session helps the learner comprehend, try again or feel a sense of satisfaction $[10,14]$. Many characteristics of graphical content can have an impact on the player's experience [20]. One of the main challenges in serious game design is in designing visuals that will to satisfy a given learning objectives. Frequently this concerns making a choice between text - to maximise the precision of the information being conveyed; and images - to achieve a more emotional effect, which is considered essential to player engagement [15]. Nelson et al. [12] found that players' memory performance when presented with different styles of images (either photographs or drawings) did not vary significantly; whereas when players were presented with text, memory performance was significantly worse as compared with images. Depending on the game mechanics, using text to convey information may have a damaging effect on a serious game's effectiveness. In addition, Lan-Ting and Kun-Chou [6] have found that learners prefer images with a determinate level of visual complexity when using small screens.

Until now, these findings related to player engagement and the use of images have not been applied to serious game design. Yet 
this research suggests that by correctly designing and managing the use of images in our designs we can improve the experience of players. Indeed, they can inform our approach to one of the key challenges in the design of serious games (particularly educational games): that of how to produce images that are effective for driving engagement. Woodrow [22] proposes a method for the design of universal health care icons. While this is a serviceable method from the point of view of a graphic designer, it fails to take into account the context for the images (e.g. the type of screen they are displayed on), user interaction, and issues relevant to designing for player engagement in a serious game.

In the absence of research into this cross-disciplinary question of how visual design affects player engagement we propose a method for designing 2D pictograms for educational serious games [10]. Below we describe how we applied this method to the design of MANTRA, a mobile game for health designed for rural Nepalese women with a low level of literacy. We conclude with a discussion of our case study and our further works

\section{BACKGROUND AND RELATED WORK}

\subsection{Engagement and graphical content}

2.1.1 The sensation of engagement. Player engagement is commonly defined using concepts such as motivation, emotion and fun [4, 15, 17]. Brockmyer et al. [1] meanwhile, associate player engagement with the sensation of presence. According to Lombard and Ditton [7], presence occurs when players consider both themselves and the game content to be sharing the same reality. For engagement and presence, the player's emotions during and after the game constitute a major factor [1, 15]. Indeed, O'Brien and Tom [15] stress affect in their definition of player engagement and Kostkova [5] studied the importance of the concept for healthcare domain. Their approach integrates concepts such as a game's aesthetics, sensory appeal and the feedback given to user.

2.1.2 Graphical content for learning. Natkin et al. [11] have shown that certain popular and highly-engaging video games can augment players' interest in scientific topics. These are video games that include scientific content represented by the game mechanics and the game's graphical content. However, graphical content cannot be defined merely by the information that it conveys. McLaughlin et al. [9] have shown that game characters in serious games are more effective for the learning process when they are depicted in an abstract as opposed to a realistic way. But player engagement also has an impact on the learning process [23] and realistic virtual environments can increase the player's sense of presence, thereby impacting player engagement $[1,7]$. Realism is defined by Lombard and Ditton [7] as the degree to which a medium produces accurate representations of objects. Abstraction is defined by McCloud [8] as the opposite of realism. He defines an abstract object as a graphic form without meaning, the visual elements of which are reduced until it retains only its essential elements. We can say, therefore, that the player's emotions, sense of enjoyment, and the game's aesthetics and success of its visual feedback all appear to be dependant on the level of player engagement, and this engagement is in turn dependant on the style of the game's graphical content (realism and abstraction). We can incorporate these considerations into a framework for the graphic designer, we must first establish an adapted terminology.

\subsection{Terminology of graphic content}

2.2.1 Content composed by graphic objects. Our approach requires differentiating the graphic objects that constitute the graphic design of a game according to their complexity. To this end, we have identified three key of types of an imagery: the sign, the symbol and the pictogram. The sign is defined by Rasmussen [18] as an object that triggers specific, predefined acts or behaviors he calls "rule-based-behaviours", which involve low-level perception. The symbol is an object that triggers "knowledge-based behaviour", and appears to require more experience to be understood or perceived in order to trigger a user action. The pictogram represents complex concepts. A designer creates pictograms by combining various simple graphic objects, such as graphemes and icons $[21]^{1}$.

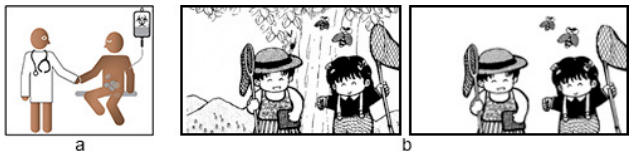

Figure 1: "Oncology" pictogram (a) [21]; high and medium level complexity (b) [6]

2.2.2 The complex pictogram paradigm. A pictogram is a more or less a stylized image that conveys complex informational content. For instance, Strauss and Zender [21] propose designing pictograms (see fig. 1-a) based on a set of concepts known by the users. They consider a pictogram to be a complex graphic object composed of elementary graphic objects such as icons and symbols. Icons, as defined by $\mathrm{Ng}$ and Chan [13] comprise both graphic features (colour, outline shape and size) and cognitive features (familiarity and meaningfulness) and can be characterized as having an visual appearance that has a certain effect on users.

Complexity has a cost, however. Some studies have found that the level of complexity of images has an impact on the users' memory performance (A "medium and low complexity" image might show characters but no background, for example.) [12, 16]. In addition, Lan-Ting and Kun-Chou [6] have found that learners prefer medium complexity images on mobile screens (see fig. 1-b). However, while these studies signal the importance in design of taking memory performance into consideration (for example, by stylizing the image or deleting the its background) they do not take into account the meaning of the image being displayed (as Strauss and Zender [21]).

In a serious game, pictograms must take account of both their meaning and their impact on the cognitive process of the player. In addition, must be "playable" in the context of the wider gameplay design and the way they contribute to the player's sense of engagement.

\footnotetext{
${ }^{1}$ According to Strauss and Zender [21], a grapheme is a graphic object that may or may not have meaning; an icon is composed of graphemes and represents a simple concept.
} 


\section{DESIGNING COMPLEX PICTOGRAMS}

We propose a novel method for designing complex pictograms for serious games that takes into account both the impact of visual complexity on memory performance and the ways in which graphical content affects player engagement. Our method comprises two complementary frameworks: one relating to graphical intentions (symbolic, aesthetic, fact) and another relating to graphical style (realism, abstraction). We envision the designer positioning their design of a pictogram somewhere in a continuum between these attributes.

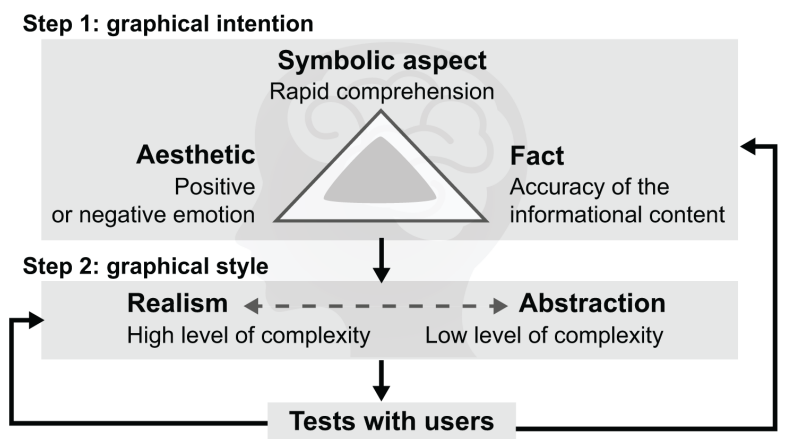

Figure 2: Method

\subsection{Step 1: graphical intention}

Figure 2 (step 01 ) shows how to emotions can be managed relative to the "quality" of the meaning and the accuracy of a pictogram's informational content. The grey area indicates the ideal positioning for a complex pictogram. Placement in this area depends on the designer's sensibilities, the intended message of the pictogram, and on the gameplay.

The term "aesthetics" in this context represents the emotional response inspired by the graphical content. According to Hunicke et al.'s three-value model for analyzing video games [3], aesthetics are the emotional responses aroused in the player during the game. This emotional element can be inspired by the graphical characteristics of a image in concert with the game mechanics and the dynamics of meaningful interaction. This "emotional part" is both an effective and a necessary factor toward achieving player engagement.

As with a game's interactive elements, symbolic elements serve to provoke player behaviors. For contexts in which the player is required to make decisions, a designer has to consider using images that draw upon the player's knowledge, as the rapid evocation of meaning is critical to ensuring smooth gameplay flow. In video games, known graphic objects or typographical signs can be used for this purpose, e.g. a question mark above the head of a quest character. Such conventions are commonly used in video games.

The "fact" component of this framework can be understood as the degree of "accuracy" used to convey information and knowledge in the game. For instance, a photograph or piece of text may be more exact or accurate than a complex pictogram but nevertheless not useful in the context of a game. "Fact" describes the serious part of a serious game.
Complex pictograms must be close to an emotional effect to improve player engagement, close to a symbolic representation for quick understanding to allow to play and close to accurate information for the serious part of the game.

\subsection{Step 2: graphical style}

Figure 2 (step 02) illustrates our second framework, "graphical style", which takes into account realism and abstraction ${ }^{2}$. These are two components that are important considerations in both in the context of player engagement and in graphic design theory [8]. For our purposes we measure realism and abstraction through the lens of complexity level, i.e. the level of visual complexity, which, as we have noted above, has an impact on memory performance.

A graphic designer must position their design between these two opposing styles considering player engagement (realism) and the learning process (abstraction). McCloud [8] emphasizes that a single image can be composed of both abstract and realistic elements. In addition, one can assume that in a serious game, the player requires more time to "read" (as opposed to understand) complex, realistic elements than more stylized ones. Finally, players tend to focus their attention on recognizable details; the designer can use this to determine how best to convey information.

\subsection{Tests with users}

In order to validate and understanding of meaning of each pictogram, the last step is to conduct tests with users. According to Strauss and Zender [21], use direct questions to know which graphic objects are useful for composing these pictograms is efficient (e.g. stethoscope for a doctor). Also, we believe these pictograms need to be evaluated in the context of the game (e.g delay of interactions, error rate). Depending on the required changes, a designer reviews the graphical intention of the pictogram (step 1: e.g the meaning is not clear - fact, users "dislike", reject the image - aesthetic) or/and adjusts the graphical style (step 2: e.g the designer adds a new graphical object).

\section{CASE STUDY: MANTRA}

The goal of MANTRA project is to use mobile technology to help increase maternal and child resilience in rural areas before, during and after disasters using mobile technology in $\mathrm{Nepal}^{3}$.

\subsection{MANTRA, the mobile game}

MANTRA is a game for public health education [19]. It is designed to improve the level of health knowledge among rural Nepalese women by teaching them how to assess the level of danger posed by various situations. Because of the lack of literacy skills, the player has to make choices and progress through the game via an entirely graphical interface (as verbal cues are not used).

It is a "point and click" style of mobile game with drag-and-drop style interactions ${ }^{4}$. It features three playable categories: maternal health, neonatal health and geohazards. Figure 3 shows the game

\footnotetext{
${ }^{2}$ See section 2.1 for the definitions.

${ }^{3}$ Acknowledgment to the Global Challenge Research Fund (GCRF): the Natural Environment Research Council (NERC), the Arts \& Humanities Research Council (AHRC), the Economic \& Social Research Council (ESRC). NERC Ref.: NE/P016103/1

${ }^{4}$ The player uses her finger to initiate the interaction, then uses her finger to move the image to another part of the screen.
} 


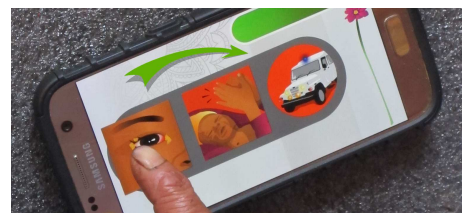

Figure 3: Second test session in Chyamrangbesi, Nepal.

interface after our test and modifications. On the left are images called "learning objective" (LO) and on the right, an image called "answer". Game difficulty is progressively increased by the inclusion of more potential responses and player progress is shown via a score bar. To start playing, the player selects what she considers to be the best LO image to match the answer and drags and drops it onto the answer image. If the player has chosen the correct LO image, she wins a point. For each successfully completed level, the player wins a flower. Overall progression is represented in terms of the number of completed flowers for each level and category.

\subsection{Applying our method}

In this section we explain how our method was applied to the design of complex pictograms to convey information and impart knowledge to the player toward the attainment of learning objectives (see Fig. 4-b).

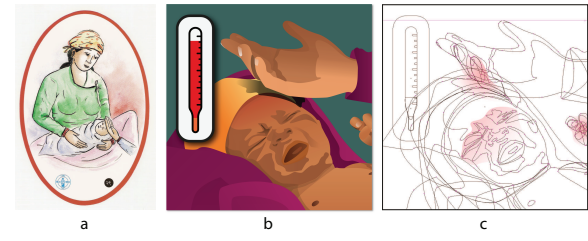

Figure 4: The baby has a fever.

4.2.1 Design workflow. The visual reference used for the creation of these illustrations produced as part of a research project ${ }^{5}$ (see Fig. 4-a) for a card game. We used the cards to inspire and inform the visual "ambience" of our game (e.g. clothes, preferred postures). We also looked at photographs taken within the target area (e.g. skin color, vegetation). First, we created sketches that we considered to be optimal, in terms of framing, positioning, size, viewing angle, composition, and so forth. Next, we created photo montages in order to determine what the "realistic" elements might be, according to our method. Finally, we drew our pictograms, using the photo montages as reference.

4.2.2 Example: designing our "baby with fever" pictogram. To give a concrete example of how we applied our method to the game MANTRA, here we describe how we designed a pictogram called "baby with fever" (see fig. 4).

Using our "graphical intentions" framework (aesthetic, symbolic, fact) we applied an "aesthetic-emotion" intention by showing a crying baby being soothed by an adult's hand. To achieve "rapid

\footnotetext{
${ }^{5}$ Mother and Infant Research Activities; funded by the Department for Internationa Development and the Institute for Global Health, London,2016.
}

symbolic meaning", we used relatively few colors, linear or circular shading and simple forms. In terms of "factual/informational content", we stayed focused on the main message. For instance, we avoided using any disruptively decorative graphic objects and we used additional element to reinforce the message e.g. a red halo on the baby's forehead. See Fig. 4-b.

To apply our "graphical style" framework (realism, abstraction) while also respecting the design choices used for the first framework, we structured the pictograms into three layers of visual elements: realistic, abstract, and the background. We included the informational content we wanted to focus on the most within the more realistic layer of visual elements. For instance, the baby's face and the mother's hand are portrayed more with more realism than the other parts of the image ${ }^{6}$ (see Fig. 4-b). We used shading, shadows and/or a larger number of vectorial points to produce this realistic layer (see Fig. 4-c and red areas). The "abstract" layer is composed of a single colour and a very small number of vectorial points. The background is a solid colour in order to lend a "medium" or "high" complexity to this pictogram [6].

4.2.3 The background. We varied the background color of the pictograms in order to limit the potential "appearance association" effect of the pictogram representing the correct answer. For 24 pictograms we used 10 randomly chosen colored backgrounds. We assumed that this system would contribute to an interface that was more visually dynamic and would focus the player's attention on the meaning of the pictogram.

\subsection{Tests with users}

4.3.1 Participants and method. We visited communities in Chandenimandan, Chyamrangbesi and Siddhipur in Nepal for the first session test. A total of 34 women participated in these interviews discussing the pictograms in Nepalese. Firstly, each pictogram was shown to participants on a paper (in pairs or individually, depending on group sizes). After observing their reaction, they were asked to define the meaning of the images (e.g. "what do you recognize?", "What do you think is on this image?"). Depending on the responses, correct meaning was explained. Then, participants were asked what graphical objects could be added or deleted to improve the image. Each interview covering 28 pictograms took about 30 - 40 minutes. All the comments were noted, and translated to English for the designer.
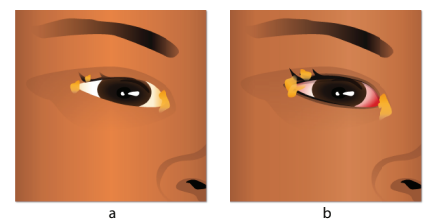

Figure 5: Before the test (a); after the modifications (b).

4.3.2 Result: an example, our design of "eyes infection". First, the participants seems to "dislike" this image - see Fig. 5-a (e.g. leave them indifferent, no particular emotion is shown). Although

${ }^{6}$ To achieve an optimum composition and viewing angle, we drew the hand of the baby in the realistic style. 
the medical case condition was recognized by most of them, we decided to reinforce the meaning of the pictogram and improve its affective aspect at the same time (step1 of our model). Our major modification was adding a red area (fact / medical symptom) and outline the eye in black ${ }^{7}$ (aesthetic). According our model and for the step 2, we used a gradient from red colour to transparent (realistic area) - see Fig. 5-b.
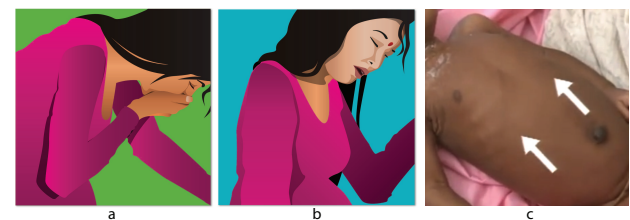

Figure 6: "vomiting" (a), "anaemia" (b), "chest indrawing" (c).

4.3.3 Discussion. Our method proved robust enough to address most of the learning objectives for our game MANTRA. Out of 28 learning objectives, only 4 could not be portrayed using our method alone. The main challenge we encountered arose when we attempted to represent diseases and symptoms such as "anaemia" (fatigue, breathlessness, pale face - see Fig. 6-b) and "chest indrawing", a symptom of pneumonia - see Fig. 6-c ${ }^{8}$. As breathlessness can be a symptom of both anaemia and pneumonia, a precise representation of chest indrawing, showing the precisely manner how the sick baby breathes, was necessary to be distinguished from other types of breathlessness.

\section{CONCLUSIONS AND FURTHER WORKS}

We propose a method for graphic design for games that enables designers to consider the impact of their design on player engagement, specifically for serious games. Our method introduces key paradigms for designing meaningful pictograms that affects player engagement into the graphic design process. Applying scientific findings about player engagement and memory performance to the design process can guide the designer's work and help the designer produce more impactful games. We have applied our method to the creation of a "visual language" used in a serious game that deals with the health risks faced by Nepalese women. Our initial results suggest that our method can directly assist designers in formalizing the main intention of each learning objective. In addition, using a bespoke method like this for conceptualizing a pictogram during the design process enhances collaboration with experts, informing the allocation of resources and shaping expectations.

For evaluating serious games, a mixed-method, cross-data analysis can be useful [4]. A next logical step could be to expand our method to include the animation process, thus widening the scope for possible representations and increasing the depth of the information able to be conveyed. Another step could be to adapt this method for the 3D design process. For example, the use of saturated, lighter colours in a $3 \mathrm{D}$ real-time engine is effective for evoking drama in a scene [2]; hence, for 3D interactive scenes, the

\footnotetext{
${ }^{7}$ The mothers outline the eyes of their babies in black in this area of Nepal.

${ }^{8}$ From "IMCI training video: identifying chest indrawing". The lower ribs of the chest are drawn in when the child takes a breath.
}

saturation level should be included in any design method for serious games in order to better manage the evocation of emotion. To improve our method, it will be necessary to evaluate the impact of its use upon the effectiveness of a serious game it is used to help design.

\section{REFERENCES}

[1] Jeanne H. Brockmyer, Christine M. Fox, Kathleen A. Curtiss, Evan McBroom, Kimberly M. Burkhart, and Jacquelyn N. Pidruzny. 2009. The development of the Game Engagement Questionnaire : A measure of engagement in video game-playing. Journal of Experimental Social Psychology 45 (2009), 624-634.

[2] Magy Seif El-Nasr. 2006. Projecting tension in virtual environments through lighting. In Proceedings of the 2006 ACM SIGCHI international conference on Advances in computer entertainment technology (ACE '06). ACM, New York, NY, USA, Article 63. https://doi.org/10.1145/1178823.1178898

[3] Robin Hunicke, Marc LeBlanc, and Robert Zubek. 2004. MDA: A formal approach to game design and game research. In Proceedings of the AAAI Workshop on Challenges in Game AI.

[4] K. Karadimitriou and M. Roussou. 2011. Studying Player Experience in a Collaborative Embodied Interaction Game. In Games and Virtual Worlds for Serious Applications (VS-GAMES), 2011 Third International Conference on. IEEE, 199-206.

[5] Patty Kostkova. 2016. User engagement with digital health technologies. In Why Engagement Matters. Springer, 127-156.

[6] Wang Lan-Ting and Lee Kun-Chou. 2014. The Study of Learners' Preference for Visual Complexity on Small Screens of Mobile Computers Using Neural Networks. TOfET: The Turkish Online fournal of Educational Technology 13, 2 (2014).

[7] Matthew Lombard and Theresa Ditton. 1997. At the Heart of It All : The Concept of Presence. Journal of Computer Mediated Communication 3 (1997).

[8] Scott McCloud. 1993. Understanding Comics : The Invisible Art. HarperCollins Publishers.

[9] Tim McLaughlin, Dennie Smith, and Irving A Brown. 2010. A framework for evidence based visual style development for serious games. In Proceedings of the Fifth International Conference on the Foundations of Digital Games. ACM, 132-138.

[10] Andreea Molnar and Patty Kostkova. 2013. On effective integration of educational content in serious games: Text vs. game mechanics. In Advanced Learning Technologies (ICALT), 2013 IEEE 13th International Conference on. IEEE, 299-303.

[11] Stephane Natkin, Delphine Soriano, Grozdana Erjavec, and Marie Durand. 2013. Could the Player's Engagement in a Video Game Increase His/Her Interest in Science? LNCS proceedings - Advances in Computer Entertainment 8253 (2013), 608-611

[12] Thomas O Nelson, Jacqueline Metzler, and David A Reed. 1974. Role of details in the long-term recognition of pictures and verbal descriptions. Fournal of Experimental Psychology 102, 1 (1974), 184.

[13] Annie Wy Ng and Alan Hs Chan. 2009. What Makes an Icon Effective? AIP Conference Proceedings 1089, 1 (2009), 104-114. https://doi.org/10.1063/1.3078113 arXiv:http://aip.scitation.org/doi/pdf/10.1063/1.3078113

[14] Heather O'Brien and Paul Cairns. 2016. Why Engagement Matters: CrossDisciplinary Perspectives of User Engagement in Digital Media. Springer.

[15] Heather L O'Brien and Elaine G Toms. 2008. What is user engagement A conceptual framework for defining user engagement with technology. Fournal of the American Society for Information Science and Technology 59, 6 (2008), 938-955.

[16] Kathy Pezdek, Ruth Maki, Debra Valencia-Laver, Tony Whetstone, Janet Stoeckert, and Tom Dougherty. 1988. Picture memory: Recognizing added and deleted details. Fournal of Experimental Psychology: Learning, Memory, and Cognition 14, 3 (1988), 468.

[17] Andrew K Przybylski, C Scott Rigby, and Richard M Ryan. 2010. A motivational model of video game engagement. Review of General Psychology 14, 2 (2010). http: //apps.isiknowledge.com.molly.ruc.dk/full_record.do?product=WOS\&search_ mode $=$ GeneralSearch\&qid $=8 \&$ SID $=$ Y2HD4jeAFEdPHIIllL $5 \&$ page $=1 \&$ doc $=1$

[18] Jens Rasmussen. 1983. Skills, rules, and knowledge; signals, signs, and symbols, and other distinctions in human performance models. Systems, Man and Cybernetics, IEEE Transactions on 3 (1983), 257-266.

[19] Ben Sawyer and Peter Smith. 2008. Serious Games Taxonomy. (Feb. 2008). http://www.seriousgames.org/presentations/serious-games-taxonomy-2008_ web.pdfhttp://fr.slideshare.net/Caspianchris/taxonomy-of-serious-games

[20] J.L. Sherry, K. Lucas, BS Greenberg, and K. Lachlan. 2006. Video game uses and gratifications as predictors of use and game preference. Playing video games. Motives, responses, and consequences (2006), 213-224.

[21] Alisa Strauss and Mike Zender. 2017. Design By Consensus: A New Method for Designing Effective Pictograms. Chapter 51.2, 7-33.

[22] Jeffery Woodrow. 2016. ULOU: Universal Language of Understanding Designing a set of universal health care icons. (2016).

[23] Gabe Zichermann. 2010. Fun is the Future: Mastering Gamification. In $h t t p: / / w w w . g a m e s f o r n a t u r e . o r g /$. 